\title{
Algorithm for Solving Fuzzy Multiobjective Linear Fractional Programming Problem by Additive Weighted Method
}

\author{
Moumita Deb \\ Department of Mathematics, \\ National Institute of Technology, Silchar \\ Silchar-788010, India
}

\author{
P.K.De \\ Department of Mathematics, \\ National Institute of Technology, Silchar \\ Silchar-788010, India
}

\begin{abstract}
In this paper attention has been paid to the study of multiobjective linear fractional programming problem (MOLFPP) by using fuzzy set theoretic approach. In this approach, MOLFPP is transformed into multiobjective linear programming problem (MOLPP) by suitable transformation. In algorithm-I, MOLFPP is transformed into MOLPP by using fuzzy set theory and the pareto optimal solution of the transformed MOLPP is obtained by applying Zimmermann's min-operator model and simplex method. Further we have used additive weighted method to modify the above approach. Algorithm-II has been presented to find the pareto optimal solution of MOLFPP by applying additive weighted method. To demonstrate the applicability of the proposed approach, one numerical example is solved to find the pareto optimal solution by applying this two algorithms.
\end{abstract}

\section{General Terms}

Multiobjective linear fractional programming problem, Additive weighted method.

\section{Keywords}

Multiobjective linear fractional programming problem, Minoperator model, Additive weighted method, Fuzzy linear programming, Fuzzy numbers.

\section{INTRODUCTION}

Linear fractional programming (LFP) problems is an important planning tool for the past decades which is applied to different disciplines like engineering, business, finance, economics, etc. Fractional programming is the ratio criteria which are often used for modelling of real life problems with one or more objective(s) such as profit-cost, inventory-sales, actual cost-standard cost, output-employees, debt-equity, etc. In many practical applications like stock problems, ore blending problems, shipping schedules problems, optimal policy for a Markovian chains, sensitivity of linear programming problem, optimization of ratios of criteria gives more insight into the situations than the optimization of each criterion [3]. Multiobjective linear fractional programming is an area of multiple criteria decision making is concerned with mathematical optimization problems involving more than one objective function to be optimized simultaneously where multiple objectives are written by fractional formulas. MOLFP is an interesting topic which has been used in production planning, financial sector, inventory management, banking sector, etc.

Charnes and Cooper [1] solved a programming problem with linear fractional funtionals by resolving it into two linear programming problems. Craven et. al [2] have given the details of LFP and its equivalence. Kornbluth et.al [11] have studied a generalized approach for solving LFP under goal programming. However, there are many methods of solving fuzzy linear fractional programming (FLFP) problems are available in the literature $[7,8,11,17,18]$. Dutta et.al [5] studied restricted class of MOLPP. Luhandjula [14] solved fuzzy approaches for multiple objective linear fractional optimization. Dutta et.al [4] have tried to modify the problem of Luhandjula's approach to solve MOLFPP. Nykowski and Zolkiewski [10] has proposed a compromise procedure for MOLFPP. Furthermore, there are a few studies $[6,12]$ on MOLFPP in recent years. By suitable transformation, Chakraborty and Gupta [15] have transformed MOLFPP to formulate an equivalent MOLPP under fuzzy set theoretic approach. We have studied the paper [15] and modify the approach by using additive weighted method. In algorithm-I, we have presented MOLFPP which is transformed into MOLPP. Using Zimmermann's min operator [9] model in the transformed MOLPP, the fuzzy model is transformed into an ordinary crisp model and the pareto optimal solution of MOLPP is obtained by applying simplex method. In algorithm-II, by using additive weighted method in the transformed MOLPP and find the pareto optimal solution of the respective problem. The paper is organised as follows:

Section 2 outlines the definitions and preliminaries of LFP, MOLFPP. In section 3, we have discussed methodology I- where MOLFPP is transformed into MOLPP by suitable transformation under fuzzy set theoretic approach. By using Zimmermann's min operator model in the transformed MOLPP, it reduces to a crisp model. From that model solution is obtained. In methodology IIwe have used additive weight method in the transformed MOLPP and from that solution is obtained. Two algorithms have been presented to find the pareto optimal solution of the MOLFPP. 
Section 4 demonstrates one numerical example to illustrate the above two algorithms. Section 5 discusses the conclusions of this paper.

\section{DEFINITION AND PRELIMINARIES}

Definition 1. Linear Fractional Programming- The general format of linear fractional programming (LFP) may be written as:

subject to the constraints:

$$
\operatorname{Max} \frac{c x+\alpha}{d x+\beta},
$$

$$
x \in S=\{x \mid A x=b, x \geq 0\}
$$

where $A \in R^{m \times n}, b \in R^{m}, x \in R^{n}, c, d \in R^{n}, \alpha, \beta \in R$ and $\mathrm{S}$ is a non-empty and bounded set.

For some values of $\mathrm{x}, d x+\beta$ may be equal to zero. For that we need to make an additional assumption that-

If $A x=b, x>0$ then $d x+\beta>0$ or $d x+\beta<0$.

For convenience, assume that LFP (1) satisfies the condition:

$$
\begin{array}{r}
x \geq 0 \text { then } \\
d x+\beta>0
\end{array}
$$

Definition 2. (Craven [3]).

The two mathematical programming problems -

(i) $\operatorname{Max} \mathrm{A}(\mathrm{x})$, subject to, $x \in \Delta$,

(ii) Max $\mathrm{B}(\mathrm{x})$, subject to, $x \in \Gamma$,

will be said to be equivalent iff there is a ono-one map q(.) of the feasible set of (i), onto the feasible set of (ii), such that $\mathrm{A}(\mathrm{x})=\mathrm{B}(\mathrm{q}(\mathrm{x}))$ for all $x \in \Delta$.

Theorem 1. Equivalence of LFP and LPP (Charnes and Cooper [1] and Craven and Mond [2]).

Assume that no point $(\mathrm{y}, 0)$ with $y \geq 0$ is feasible for the following linear programming problem (LPP):

subject to the constraints:

$$
\operatorname{Max} c y+\alpha t
$$

$$
\begin{aligned}
& d y+\beta t=1, \\
& A y-b t=0
\end{aligned}
$$

where $t \geq 0, y \geq 0, y \in R^{n}, t \in R$.

Assumption: If $A x=b, x \geq 0$ then $d x+\beta>0$ or $d x+\beta<0$, then the LFP(1) is equivalent to linear program (3).

Proof: For $\mathrm{x}$ is feasible(for LFP (1)),

define $\mathrm{q}(\mathrm{x})=(\mathrm{y}, \mathrm{t})$, where $t=(d x+\beta)^{-1}$ and $\mathrm{y}=\mathrm{tx}$.

Then $y \geq 0, t>0$,

$A y-t b=A(t x)-t b=t(A x-b)=0$,

$d x+\beta=d\left(\frac{y}{t}\right)+\beta=d y+\beta t=t(d x+\beta)=1$,

Thus, $(\mathrm{y}, \mathrm{t})$ is feasible for $\operatorname{LPP}(3)$.

Conversely, if $(\mathrm{y}, \mathrm{t})$ is feasible for $\operatorname{LPP}(3)$, then $x=\frac{y}{t}, t>0$ satisfies

$x \geq 0, A x-b=A\left(\frac{y}{t}\right)-b=\frac{A y-b t}{t}=0$.

Consequently, q(.) maps the feasible set of LFP(1), one-one, onto the feasible set for LPP(3).

Also the objective function are related by

$\frac{c x+\alpha}{d x+\beta}=\frac{c(y / t+\alpha)}{d(y / t)+\beta}=\frac{c y+\alpha t}{d y+\beta t}=\frac{c y+\alpha t}{1}=(c y+\alpha t)$.

Thus, $\operatorname{LFP}(3)$ is equivalent to $\operatorname{LPP}(1)$.

\subsection{Concave-Convex Problems(Craven [3]).}

Consider the fractional programming problem

$$
\operatorname{Max} \frac{N(x)}{D(x)}
$$

subject to the constraints :

$$
\begin{gathered}
A x \leq b, x \geq 0 \text { and } \\
x \in \Delta=\{x: A x \leq b, x \geq 0\} \\
\Rightarrow D(x)>0
\end{gathered}
$$

Now consider

subject to the constraints:

$$
\operatorname{Max} t N\left(\frac{y}{t}\right)
$$

$$
\begin{aligned}
A\left(\frac{y}{t}\right)-b & \leq 0, \\
t D\left(\frac{y}{t}\right) & =1
\end{aligned}
$$

where $t>0, y \geq 0$

and $\operatorname{Max} t N\left(\frac{y}{t}\right)$

subject to the constraints:

$$
\begin{array}{r}
A\left(\frac{y}{t}\right)-b \leq 0, \\
t D\left(\frac{y}{t}\right) \leq 1 \\
\text { where } t>0, y \geq 0
\end{array}
$$

where (5) is obtained from (4) by substituting $t=\frac{1}{D(x)}, y=t x$. Equation (5) and (6) are obtained by replacing the equality constraint $t D\left(\frac{y}{t}\right)=1$ by an inequality constraint $t D\left(\frac{y}{t}\right) \leq 1$.

Definition 3. (Craven [3]).

The fractional programming problem (4) will be said to be standard concave-convex fractional programming problem (SCCFP) if $\mathrm{N}($.)is concave on $\Delta$ with $N(\xi) \geq 0$ for some $\xi \in \Delta$ and $\mathrm{D}($.) is convex and positive on $\Delta$

Theorem 2. (Schaible $[18,19]$ ).

Let for some $\xi \in \delta, N(\xi) \geq 0$, if (4) reaches a (global) maximum at $x=x^{*}$, then (6) reaches a (global) maximum at a point $(t, y)=\left(t^{*}, y^{*}\right)$ where $\frac{y^{*}}{t^{*}}=x^{*}$ and the objective functions at these points are equal.

Theorem 3. (Schaible [19]).

If (4) is a SCCFP which reaches a (global) maximum at a point $x^{*}$, then the corresponding transformed problem (6) attains the same maximum value at a point $\left(t^{*}, y^{*}\right)$, where $\frac{y^{*}}{t^{*}}=x^{*}$. Also (6) has a concave objective function and a convex feasible set.

If instead in (4), $N($.$) is concave, \mathrm{D}($.$) is concave and positive on \Delta$ and $\mathrm{N}($.$) is negative for each x \in \Delta$ then

$$
\operatorname{Max}_{x \in \Delta} \frac{N(x)}{D(x)} \Leftrightarrow \operatorname{Min}_{x \in \Delta} \frac{-N(x)}{D(x)} \Leftrightarrow \operatorname{Max}_{x \in \Delta} \frac{D(x)}{-N(x)},
$$

where $-\mathrm{N}(\mathrm{x})$ is convex and positive.

Now, by Theorem 3 and under the present hypothesis, the fractional programming (4) transformed to the following LPP :

$$
\operatorname{Max} t D\left(\frac{y}{t}\right)
$$

subject to the constraints: 


$$
\begin{array}{r}
A(y / t)-b \leq 0, \\
-t N(y / t) \leq 1 \\
\text { where } t>0, y \geq 0 .
\end{array}
$$

Definition 4. Multiobjective Linear Fractional Programming Problem:

The general format of a multiobjective linear fractional programming which is stated as follows-

subject to the constraints:

$$
\operatorname{Max} \mathrm{Z}(\mathrm{x})=\left\{Z_{1}(x), Z_{2}(x), \ldots . ., Z_{p}(x)\right\}
$$

$$
\begin{aligned}
& \qquad x \in \Delta=\left\{x \in R^{n}: A x\left(\begin{array}{c}
\leq \\
= \\
\geq
\end{array}\right) b, x \geq 0\right\} \\
& \text { with } b \in R^{n}, A \in R^{m \times n} \text { and } \\
& \qquad Z_{p}(x)=\frac{c_{p} x+\alpha_{p}}{d_{p} x+\beta_{p}}=\frac{N_{p}(x)}{D_{p}(x)} \\
& \text { where } c_{p}, d_{p} \in R^{n} \text { and } \alpha_{p}, \beta_{p} \in R .
\end{aligned}
$$

\section{METHODOLOGY}

\subsection{Methodology-I}

3.1.1 Transformation of MOLFPP to MOLPP. Max $Z(x)=$ $\left\{Z_{1}(x), Z_{2}(x)\right.$

$$
\left.Z_{p}(x)\right\}
$$

Take $Z_{1}(x)=\frac{N_{1}(x)}{D_{1}(x)}=\frac{P_{1} x_{1}+Q_{1} x_{2}}{R_{1} x_{1}+S_{1} x_{2}+T_{1}}=P_{1} y_{1}^{\prime}+Q_{1} y_{2}^{\prime}$

where $t=\frac{1}{R_{1} x_{1}+S_{1} x_{2}+T_{1}}$ and $y_{1}^{\prime}=t x_{1}, y_{2}^{\prime}=t x_{2}$.

$Z_{2}(x)=\frac{N_{2}(x)}{D_{2}(x)}=P_{2} y_{1}^{\prime \prime}+Q_{2} y_{2}^{\prime \prime}$

where $t=\frac{1}{R_{2} x_{1}+S_{2} x_{2}+T_{2}}$ and $y_{1}^{\prime \prime}=t x_{1}, y_{2}^{\prime \prime}=t x_{2}$.

Similarly, $Z_{3}(x)=\frac{N_{3}(x)}{D_{3}(x)}=P_{3} y_{1}^{\prime \prime \prime}+Q_{3} y_{2}^{\prime \prime \prime}$

$$
Z_{p}(x)=\frac{N_{p}(x)}{D_{p}(x)}=P_{p} y_{1}^{\prime p}+Q_{p} y_{2}^{\prime p}
$$

subject to the constraints:

$$
\begin{gathered}
x \in \Delta=\left\{x \in R^{n}: A x\left(\begin{array}{l}
\leq \\
= \\
\geq
\end{array}\right) b, x \geq 0\right\} \\
\text { with } b \in R^{n}, A \in R^{m \times n}
\end{gathered}
$$

which can be written as-

$$
A_{1} x_{1} \leq b_{1} \Rightarrow A_{1} y_{1}^{\prime}-b_{1} t \leq 0,
$$

Similarly, $A_{2} y_{1}^{\prime \prime}-b_{2} t \leq 0$,

$$
\begin{gathered}
A_{p} y_{1}^{p}-b_{p} t \leq 0, \\
t\left(R_{1} x_{1}+S_{1} x_{2}+T_{1}\right)=1 \\
t\left(R_{2} x_{1}+S_{2} x_{2}+T_{2}\right)=1 \\
\ldots \ldots \ldots \ldots \ldots . . . \\
t\left(R_{p} x_{1}+S_{p} x_{2}+T_{p}\right)=1
\end{gathered}
$$

where $y_{1}^{\prime}, y_{2}^{\prime}, y_{1}^{\prime \prime}, y_{2}^{\prime \prime}, \ldots \ldots . ., y_{1}^{\prime}, y_{2}^{\prime p}, t, x_{1}, x_{2} \geq 0$.

The given MOLFPP is equivalent to the following MOLPP as follows:

$\operatorname{Max}\left\{f_{1}(y, t)=P_{1} y_{1}^{\prime}+Q_{1} y_{2}^{\prime}, f_{2}(y, t)=P_{2} y_{1}^{\prime \prime}+\right.$ $\left.Q_{2} y_{2}^{\prime \prime}, \ldots \ldots \ldots, f_{p}(y, t)=P_{p} y_{1}^{\prime p}+Q_{p} y_{2}^{\prime p}\right\}$ subject to the constraints:

$$
\begin{aligned}
& A_{1} y_{1}^{\prime}-b_{1} t \leq 0, \\
& A_{2} y_{1}^{\prime \prime}-b_{2} t \leq 0,
\end{aligned}
$$

$$
A_{p} y_{1}^{\prime p}-b_{p} t \leq 0,
$$

$$
\begin{aligned}
& t\left(R_{1} x_{1}+S_{1} x_{2}+T_{1}\right)=1 \text {, } \\
& t\left(R_{2} x_{1}+S_{2} x_{2}+T_{2}\right)=1 \text {, } \\
& t\left(R_{p} x_{1}+S_{p} x_{2}+T_{p}\right)=1,
\end{aligned}
$$

where $y_{1}^{\prime}, y_{2}^{\prime}, y_{1}^{\prime \prime}, y_{2}^{\prime \prime}, \ldots \ldots . ., y_{1}^{\prime p}, y_{2}^{\prime p}, t, x_{1}, x_{2} \geq 0$.

Solving the above problem we get the results of $f_{1}(y, t), f_{2}(y, t), \ldots \ldots \ldots, f_{p}(y, t)$.

3.1.2 Fuzzy Approach to MOLFPP and its Crisp Solution.

Let I be the index set such that

$$
I=\left\{i: N_{i}(x) \geq 0 \text { for some } x \in S\right\} \text { and }
$$$$
I^{c}=\left\{i: N_{i}(x)<0 \text { for each } x \in S\right\} \text { where }
$$

$I \cup I^{c}=1,2, \ldots ., n$.

Let $\mathrm{D}($.) be positive on $\mathrm{S}$ where $\mathrm{S}$ is non-empty and bounded.

Let the least value of $\frac{1}{D_{i}(x)}$ and $\frac{1}{-N_{i}(x)}$ is $\mathrm{t}$. i.e.,

$$
\cap_{i \in I} \frac{1}{D_{i}(x)}=t
$$

and

$$
\cap_{i \in I^{c}} \frac{1}{-N_{i}(x)}=t
$$

which is equivalent to-

$$
\frac{1}{D_{i}(x)} \geq t \quad \text { for } \quad i \in I
$$

and

$$
\frac{1}{-N_{i}(x)} \geq t \quad \text { for } \quad i \in I^{c}
$$

Using the transformation $\mathrm{y}=\mathrm{tx}(t>0)$ and by inequalities (10), an equivalent MOLPP for MOLFPP may be written as-

$\operatorname{Max}\left\{t N_{i}(y / t)\right.$, for $\quad i \in I ; \quad t D_{i}(y / t)$, for $\left.i \in I^{c}\right\}$ subject to the constraints:

$$
\begin{aligned}
& \quad t D_{i}(y / t) \leq 1 \quad \text { for } \quad i \in I \\
& -t N_{i}(y / t) \leq 1 \quad \text { for } \quad i \in I^{c} \\
& A(y / t)-b \leq 0 \\
& \text { where } t \geq 0, y \geq 0 \text {. }
\end{aligned}
$$

Using Zimmermann's min operator [9], the fuzzy model (11) transformed to the crisp model as follows:

$$
\operatorname{Max} \lambda
$$

subject to the constraints :

$$
\begin{gathered}
\mu_{i}\left(t N_{i}(y / t)\right) \geq \lambda \text { for } i \in I, \\
\mu_{i}\left(t D_{i}(y / t)\right) \geq \lambda \text { for } i \in I^{c}, \\
t D_{i}(y / t) \leq 1 \text { for } i \in I,
\end{gathered}
$$




$$
\begin{aligned}
& \quad-t N_{i}(y / t) \leq 1 \quad \text { for } \quad i \in I^{c}, \\
& A(y / t)-b \leq 0 \text {, } \\
& \text { where } y \geq 0, t \geq 0 \text {. }
\end{aligned}
$$

For $i \in I$, the membership function is:

$$
\mu_{i}\left(t N_{i}(y / t)\right)=\left\{\begin{array}{ccc}
0 & \text { if } t N_{i}(y / t) \leq 0 \\
\frac{t N_{i}(y / t)-0}{\check{Z}_{i}-0} & \text { if } 0<t N_{i}(y / t) \leq \check{Z}_{i} \\
1 & \text { if } t N_{i}(y / t) \geq \check{Z}_{i}
\end{array}\right.
$$

and for $i \in I^{c}$, the membership function is:

$$
\mu_{i}\left(t D_{i}(y / t)\right)=\left\{\begin{array}{ccc}
0 & \text { if } t D_{i}(y / t) \leq 0 \\
\frac{t D_{i}(y / t)-0}{\check{Z}_{i}-0} & \text { if } 0<t D_{i}(y / t) \leq \check{Z}_{i} \\
1 & \text { if } t D_{i}(y / t) \geq \check{Z}_{i}
\end{array}\right.
$$

\subsection{Algorithm}

3.2.1 Algorithm-I. Attention has been paid to compute the solution of MOLFPP. Algorithm-I for finding optimal solution of MOLFPP can be summerized in a series of steps which are as follows:-

Step 1: First convert each objective function of MOLFPP to MOLPP by using transformation $\mathrm{y}=\mathrm{tx}(t>0)$.

Step 2: Convert each constraint by substituting $y=t x$.

Step 3: The above problem reduces to the following:

$$
\operatorname{Max}\left\{f_{1}(y, t), f_{2}(y, t), \ldots \ldots \ldots, f_{p}(y, t)\right\}
$$

subject to the constraints:

$$
\begin{aligned}
& A_{1} y_{1}^{\prime}-b_{1} t \leq 0, \\
& A_{2} y_{1}^{\prime \prime}-b_{2} t \leq 0 \text {, } \\
& A_{p} y_{1}^{p}-b_{p} t \leq 0 \\
& t\left(R_{1} x_{1}+S_{1} x_{2}+T_{1}\right)=1, \\
& t\left(R_{2} x_{1}+S_{2} x_{2}+T_{2}\right)=1 \text {, } \\
& t\left(R_{p} x_{1}+S_{p} x_{2}+T_{p}\right)=1
\end{aligned}
$$

where $y_{1}^{\prime}, y_{2}^{\prime}, y_{1}^{\prime \prime}, y_{2}^{\prime \prime}, \ldots \ldots . ., y_{1}^{\prime p}, y_{2}^{\prime p}, t, x_{1}, x_{2} \geq 0$.

Step 4: Apply simplex method to find the values of $f_{1}(y, t), f_{2}(y, t), \ldots \ldots \ldots, f_{p}(y, t)$.

Step 5: Determine the membership functions of each objective which are $\mu_{i}\left(t N_{i}(y / t)\right)$ for $i \in I$ and $\mu_{i}\left(t D_{i}(y / t)\right)$ for $i \in I^{c}$.

Step 6: Apply Zimmermann's min operator [9] to transform the fuzzy model (10) can be transformed to the crisp one as follows: $\operatorname{Max} \lambda$

subject to the constraints:

$$
\begin{gathered}
\mu_{i}\left(t N_{i}(y / t)\right) \geq \lambda \text { for } i \in I, \\
\mu_{i}\left(t D_{i}(y / t)\right) \geq \lambda \text { for } i \in I^{c} \\
t D_{i}(y / t) \leq 1 \text { for } i \in I, \\
-t N_{i}(y / t) \leq 1 \text { for } i \in I^{c} \\
A(y / t)-b \leq 0 \\
\text { where } y \geq 0, t \geq 0 .
\end{gathered}
$$

Step 7: Apply standard LP package to find the values of $y_{1}, y_{2}, \ldots \ldots \ldots, y_{p}, t, \lambda$.

Step 8: Convert the above values $y_{1}, y_{2}, \ldots \ldots ., y_{p}, t, \lambda$ by using the transformation $\mathrm{y}=\mathrm{tx}$ and then substitute the values in the given equation.

Step 9: Using the values of $x_{1}, x_{2}, \ldots ., x_{p}$ compute the values of
$Z_{1}, Z_{2}, \ldots \ldots, Z_{p}$

\subsection{Methodology-II}

\subsubsection{Weighted Additive Method to Solve Multiobjective Fuzzy Linear Fractional Programming Problem}

Chen et.al [13] have used weighted average method in "Fuzzy goal programming with different importance and properties". Tiwari, Dharmar and Rao [16] have mentioned an additive model in fuzzy goal programming which incorporates each goal's weight $W_{k}$ into the corresponding objective function

$$
\text { i.e., } Z=\sum_{k=1}^{n} W_{k} Z_{k}
$$

where $Z_{k}$ denotes the kth fuzzy goal and

$$
\sum_{k=1}^{n} W_{k}=1
$$

In the additive model weights show the relative importance of the goals. Now for simplicity, the importance of these objectives (goals) are taken to be different. Hence all objective function can be reformulated as a single objective function without adding any more constraints.

$$
\operatorname{Max} \quad Z=W_{1} Z_{1}+W_{2} Z_{2}+\ldots \ldots \ldots \ldots+W_{p} Z_{p}
$$

such that under given constraints,

where

$$
\sum_{k=1}^{p} W_{k}=1
$$

3.3.2 Algorithm-II. Algorithm for finding the solution of MOLFPP by additive weighted method. The suggested algorithm-II can be summarized in the following way:-

Step 1: Step 1 to step 3 to be followed from algorithm-I. Step 4: Apply additive weighted method:

$$
\operatorname{Max} \quad Z=W_{1} f_{1}(y, t)+W_{2} f_{2}(y, t)+\ldots \ldots \ldots \ldots+W_{p} f_{p}(y, t)
$$

such that under given constraints, where

$$
\sum_{k=1}^{p} W_{k}=1
$$

Step 5: Apply standard LP package to find the values of $y_{1}, y_{2}, \ldots \ldots \ldots, y_{p}, t$.

Step 6: Convert the above values $y_{1}, y_{2}, \ldots \ldots \ldots, y_{p}, t$ by using the transformation $\mathrm{y}=\mathrm{tx}$ and then obtain the values of $Z_{1}, Z_{2}, \ldots \ldots ., Z_{p}$.

\section{NUMERICAL EXAMPLE}

\subsection{Example 1}

The following numerical example studied by Luhandjula [14] is considered to illustrate the above approach: 
Consider a MOLFPP with two objective functions as follows:

$\operatorname{Max} Z(x)=\left(Z_{1}(x)=\frac{x_{1}-4}{-x_{2}+3}, Z_{2}(x)=\frac{-x_{1}+4}{x_{2}+1}\right)$

subject to the constraints:

$$
\begin{gathered}
-x_{1}+3 x_{2} \geq 0, \\
x_{1} \leq 6,
\end{gathered}
$$

Solution:

$$
\text { where } x_{1}, x_{2} \geq 0 \text {. }
$$

Here, $Z_{1}(x)>0$ for some $\mathrm{x}$ in the feasible region and $Z_{2}(x) \leq 0$ for each $\mathrm{x}$ in the feasible region.

The above MOLFPP is equivalent to the following MOLPP-

$\operatorname{Max}\left\{f_{1}(y, t)=y_{1}-4 t, f_{2}(y, t)=-y_{1}+4 t\right\}$

subject to the constraints:

$$
\begin{aligned}
& y_{2}+t \leq 1, \\
& -y_{1}+4 t \leq 1 \\
& -y_{1}+3 y_{2} \leq 0 \\
& y_{1}-6 t \leq 0 \\
& -y_{2}+3 t=1 \\
& y_{1}, y_{2}, t \geq 0
\end{aligned}
$$

After solving the problem we get, $f_{1}(y, t)=1$ and $f_{2}(y, t)=$ 0.99 .

Using the membership functions defined in (13) and (14), the above multiobjective linear programming problem reduces to the crisp model as follows:

subject to the constraints:

$$
\operatorname{Max} \lambda
$$

$$
\begin{gathered}
-y_{1}+3 t-\lambda \geq 0, \\
-1.01 y_{1}+4.04 t-\lambda \geq 0, \\
-y_{2}+t \leq 1, \\
-y_{1}+4 t \leq 1, \\
-y_{1}+3 y_{2} \leq 0, \\
y_{1}-6 t \leq 0, \\
-y_{2}+3 t=1, \\
y_{1}, y_{2}, t, \lambda \geq 0 .
\end{gathered}
$$

After solving the above problem by LP package, we get,

$$
y_{1}=0.33, y_{2}=0, t=0.33 \text { and } \lambda=0.67 \text {. }
$$

the pareto optimal solution of the given problem is :

$$
x_{1}=1, x_{2}=0, Z_{1}(x)=-\frac{4}{3}, Z_{2}(x)=3 \text {. }
$$

\subsection{Example 1:. Multiobjective fuzzy linear fractional pro-} gramming formulation of example 1:

The above MOLFPP is equivalent to the following MOLPP -

$\operatorname{Max}\left\{f_{1}(y, t)=y_{1}-4 t, f_{2}(y, t)=-y_{1}+4 t\right\}$

subject to the constraints:

$$
\begin{aligned}
& y_{2}+t \leq 1, \\
& -y_{1}+4 t \leq 1, \\
& -y_{1}+3 y_{2} \leq 0 \\
& y_{1}-6 t \leq 0, \\
& -y_{2}+3 t=1, \\
& y_{1}, y_{2}, t \geq 0
\end{aligned}
$$

Solution:

Substituting $W_{1}=0.8, W_{2}=0.2$ and $f_{1}(y, t)=y_{1}-4 t, f_{2}(y, t)=-y_{1}+4 t$ in (19), we get
$\operatorname{Max} Z=W_{1} f_{1}(y, t)+W_{2} f_{2}(y, t)=0.6 y_{1}-2.4 t$

subject to the constraints:

$$
\begin{aligned}
& y_{2}+t \leq 1, \\
& -y_{1}+4 t \leq 1, \\
& -y_{1}+3 y_{2} \leq 0 \\
& y_{1}-6 t \leq 0 \\
& -y_{2}+3 t=1, \\
& y_{1}, y_{2}, t \geq 0
\end{aligned}
$$

After solving the problem by simplex method we get,

$$
y_{1}=3, y_{2}=0.50 \text { and } t=0.50 \text {. }
$$

$$
\operatorname{Max} \quad Z=0.60 \text {. }
$$

The pareto optimal solution of the MOLFPP is as follows:

$$
x_{1}=6, x_{2}=1, Z_{1}(x)=1, Z_{2}=-1 .
$$

\section{CONCLUSIONS}

Here attention has been paid to the study of the pareto optimal solution of MOLFPP. Two algorithms has been proposed for presenting our approach. Algorithm-I gives the steps of transforming a MOLFPP into MOLPP by applying suitable transformation and Zimmermann's min-operator model. Later on it has been solved by using some standard LP package. Algorithm-II is followed from algorithm-I after first 3 steps. Then additive weighted method has been applied to transform MOLPP to single objective linear programming problem and then it has been solved by standard LP package. The proposed approach to solve MOLFPP yields an efficient solution which reduces the complexity in problem solving and it is easy to compute.

\section{REFERENCES}

[1] Charnes, A. and Cooper,W.W. 1962. Programming with linear fractional functionals, Naval Research Logistics Quart., 9, 181-186

[2] Craven, B.D. and Mond, B. 1975. On fractional programming and equivalence, Naval Research Logistics Quar., 22, 405-410.

[3] Craven, B. D. 1988. Fractional programming, Heldermann Verlag, Berlin, 145PP, DM-48, Sigma Series in Applied Mathematics, 4.

[4] Dutta,D., Rao, J.R. and Tiwari,R.N. 1992. Multiple objective linear fractional programming-A fuzzy set theoretic approach, Fuzzy Sets and Systems, 52, 39-45.

[5] Dutta,D., Rao,J.R. and Tiwari, R.N. 1993. A restricted class of multiobjective linear programming problems, European Journal Operations Research, 68, 352-355.

[6] Dutta,D., Rao, J.R. and Tiwari,R.N. 1993. Fuzzy approaches for multiple criteria linear fractional optimization:a comment, Fuzzy Sets and Systems, 54, 347-349.

[7] Munteanu, E. and Rado,F. 1960. Calculul sarjelor celor mai economice la cuptoarele de topit fonta, studii si cercetari matematice, cluj, faseiola anexa, XI, 149-158. 
[8] Choo, E.U. and Atkins,D.R. 1982. Bicriteria linear fractional programming, Journal Optimization Theory Application, 36, 203-220.

[9] Zimmermann, H.J. 1976. Description and optimization of fuzzy systems, International Journal General Systems, 2, 209-215.

[10] Nykowski, I. and Zolkiewski,Z. 1985. A compromise procedure for the multiple objective linear fractional programming problem, European Journal Operation Research, 19, 91-97.

[11] Kornbluth, J.S.H. and Steuer, R.E. 1981. Goal programming with linear fractional criteria, European Journal Operation Research, 8, 58-65.

[12] Kornbluth, J.S.H. and Steuer, R.E. 1981. Multiple objective linear fractional programming, Management Science, 27, 1024-1039.

[13] Lin, K. and Chen, M. 1994. The fuzzy shortest path problem and its most vital areas, Fuzzy Sets and Systems, vol.58, 343-353.

[14] Luhandjula, M.K. 1984. Fuzzy approaches for multiple objective linear fractional optimization, Fuzzy Sets and Systems, 13, 11-23.

[15] Chakraborty, M. and Gupta, S. 2002. Fuzzy mathematical programming for multi-objective linear fractional programming problem, Fuzzy Sets and Systems, 125, 335-342.

[16] Tiwari, R.N., Dharmar,S. and Rao,J.R. 1987. Fuzzy goal programming-an additive model, Fuzzy sets and Systems, 24, 27-34.

[17] Zionts, S. 1968. Programming with linear fractional functionals, Naval Research Logistics Quart., 15, 449-451.

[18] Schaible, S.1976. Fractional programming I: duality, Management Science, A 22, 658-667.

[19] Schaible, S. 1978. Analyse and Anwendungen von Quotientnprogrammen, Verlag anton Hain, Meisenheim am Glan. 